\title{
The Use of a Wound Protector as a Self Retaining Skin Retractor for Skin Sparing Mastectomy and Immediate Reconstruction
}

\section{Chaturvedi S* and Ramsay G}

Aberdeen Royal Infirmary, Foresterhill, Aberdeen AB252ZN, UK

\begin{abstract}
Introduction: The skin sparing mastectomy allows appropriate skin closure for immediate reconstruction without threatening the oncological intent of the operation. Obtaining access to the peripheral aspects of the breast through a circum-areolar or a small incision can be difficult. Conventional retraction with metallic retractors leads to extensive point pressure on the skin flaps leading potentially to skin necrosis. Flexible Ring wound protectors are now commonplace in abdominal procedures. Here, we describe the use of the Alexis ${ }^{\circledR}$ wound retractor (Applied Medical, USA) in skin sparing mastectomies.

Methodology and technique: The incision is a limited elliptical incision including the nipple-areolar complex. Skin flaps are raised circumferentially to approximately $3 \mathrm{~cm}$ all round and the internal ring of the medium sized Alexis ${ }^{\circledR}$ wound retractor $(2.5$ to $6 \mathrm{~cm}$ ) is placed within the wound. The external component of the device is rolled inward until sufficient retraction is obtained. The dissection plane is easily visualised through the circumferential retraction obtained from the wound retractor with little additional conventional retraction and the skin sparing mastectomy is undertaken. We have also found this product helpful in axillary dissection placing small $(2.5 \mathrm{~cm})$ retractor with in the medium one.

Discussion: We have used Alexis ${ }^{\circledR}$ retractor (Figure 1) in 30 skin sparing mastectomies and 16 axillary dissections. We have found this to be particularly useful as a retractor as the dissection plane is easily visualised. The device also provides wound protection from potential infective processes in an environment where prosthetic material is likely to be placed. The circumferential retraction allows appropriate exposure in both the breast and axilla without compromising blood supply of skin flap.
\end{abstract}

Keywords: Mastectomy; Wound protector; Skin; Retractor

\section{Introduction}

Initially described in 1962, the skin sparing mastectomy was first used for benign lesions [1]. It subsequently took thirty years before this procedure was undertaken for malignant disease $[2,3]$. Since then, with proven improved aesthesis [4] and psychological outcome [5] it has become a common operation for those women who wish to undergo reconstruction.

Breast reconstruction at the same time as mastectomy (immediate breast reconstruction) has also been adopted in an attempt to minimise the psychological trauma and reducing the stigma associated with whole breast loss. However, whether it achieves these aims remain unclear [6]. Immediate breast reconstruction operations do not impact on survival outcome [7] nor do they compromise adjuvant chemotherapy delivery [8] when compared to standard mastectomy or delayed reconstruction.

Immediate breast reconstruction operations are unfortunately not without a significant number of post operative complications. Craft et al. [9] describe the immediate breast reconstruction as having one of the highest infection related complications in the plastic surgery

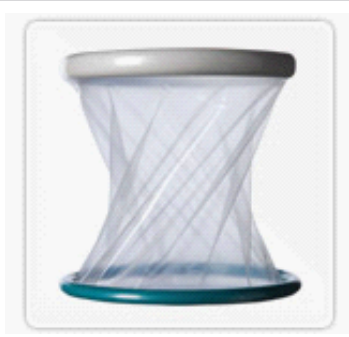

Figure 1: Alexis ${ }^{\circledR}$ Retractor (Curtsey of Applied Medical, USA). specialty. This is despite the breast surgery being a clean procedure, with no bacterial contamination during the operation. Wound infection, flap necrosis and implant infection are among the most common complications. In Lower GI procedures which often lead to exposure of bacterial loaded contaminants the wound protectors are commonplace and have had marked reductions in the prevalence of wound infections from $20 \%$ to $<10 \%$ [10].

One such wound protector is the Alexis ${ }^{\circledR}$ retractor (Applied Medical, USA). This has become a frequently used equipment in laparoscopic anterior resection wherein big specimen needs to be delivered through a smaller incision akin to skin sparing mastectomy, due to its added advantage of being able to assist in retraction [11]. It is available in different sizes, large, medium and small [12]. To our knowledge, it is infrequently used for breast surgery. In this paper, we describe our experiences of its use in the skin sparing mastectomy of all 5 varieties $[13,14]$.

\section{Operative and Technical Experience}

All breast cancer management decisions are made at a weekly

*Corresponding author: Shailesh Chaturvedi, Consultant Oncoplastic Breast Surgeon, Aberdeen Royal Infirmary, Aberdeen, AB252ZN, UK, Tel: 01224554466 E-mail: s.chaturvedi@abdn.ac.uk; s.chaturvedi@nhs.net

Received March 05, 2013; Accepted April 10, 2013; Published April 15, 2013

Citation: Chaturvedi S, Ramsay G (2013) The Use of a Wound Protector as a Self Retaining Skin Retractor for Skin Sparing Mastectomy and Immediate Reconstruction. Trop Med Surg 1: 112. doi:10.4172/tpms.1000112

Copyright: (c) 2013 Chaturvedi S, et al. This is an open-access article distributed under the terms of the Creative Commons Attribution License, which permits unrestricted use, distribution, and reproduction in any medium, provided the original author and source are credited. 
Citation: Chaturvedi S, Ramsay G (2013) The Use of a Wound Protector as a Self Retaining Skin Retractor for Skin Sparing Mastectomy and Immediate Reconstruction. Trop Med Surg 1: 112. doi:10.4172/2329-9088.1000112

Page 2 of 3

multidisciplinary meeting. The decision for immediate reconstruction takes into account the pathological, radiological, patient morphology and other surgical factors as well as the wishes of the patient.

\section{Technique}

The incision used is a limited elliptical incision encompassing the nipple-areolar complex or circumareolar when nipple areolar complex has to be sacrificed. Skin flaps are raised circumferentially in subcutaneous plane to approximately $3 \mathrm{~cm}$ all round using skin hook retractors. The internal ring of the medium sized Alexis ${ }^{\circledR}$ wound protector/retractor is then placed within the wound. The external component of the device is rolled inward until retraction is achieved. The dissection plane is easily visualised through the circumferential retraction obtained from the wound retractor (Figures 2 and 3 ).

These retractors have been in common usage in the General Surgical field and therefore have been through the appropriate pre use safety checks and no further safety review was deemed necessary.

Further retraction may be required with either the assistant's finger or light pressure with a standard metallic retractor (Figure 4). The skin sparing mastectomy is performed in a standard manner, ensuring that all breast tissue is excised from the inferior border of the clavicle to the infra-mammary fold. The specimen can then be removed through the

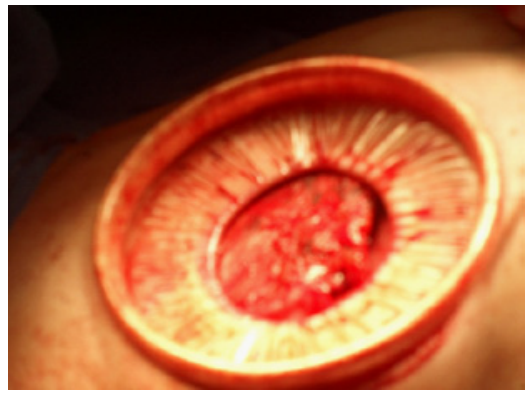

Figure 2: Circumferential retraction

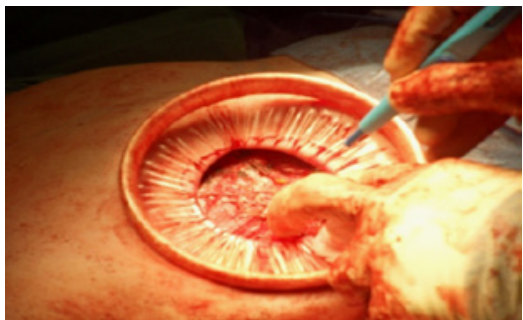

Figure 3: Alexis ${ }^{\circledR}$ Retractor/Protector in Use.

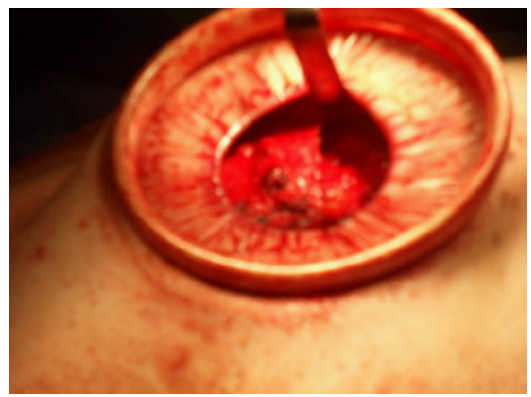

Figure 4: Minimal retraction with metallic retractor.

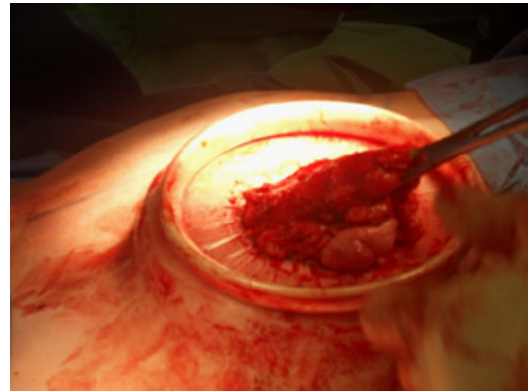

Figure 5: Delivering the resected breast through Alexis ${ }^{\circledR}$ retractor.

retractor (Figure 5) and, once haemostasis has been achieved, a wash of the mastectomy bed can then be undertaken.

Immediate reconstruction can then be undertaken as planned. There are no differences in closure to our previous techniques.

\section{Results and Outcomes}

We have now used this retractor in 30 mastectomies, ( 7 bilateral and 16 unilateral mastectomies). Our infection rate (taken as any redness, swelling, discharge to the point of review at outpatient clinic 6 weeks post operatively) has dropped from $>10 \%$ to $<2 \%$ since we started using Alexis ${ }^{\circledR}$ retractor.

We have found that the use of this device leads to comparatively little additional conventional retraction being necessary. As a result, we have also used this retractor/wound protector in axillary clearances by placing a smaller sized device within the wound. Again, we have found this to be helpful, both in terms of infection control and retraction. We have used the device in 16 axillary clearances.

\section{Discussion}

Risk factors associated with adverse surgical outcomes include, increased BMI and larger breast volume $[3,12]$. Hypotheses for the latter have included more extensive skin flaps and, as a result, a more compromised sub dermal arterial plexus. By limiting the skin incision to allow for as much skin as possible to be spared for subsequent reconstruction, the operative view is inevitably compromised in comparison to a standard mastectomy. This leads to more extensive retraction from the assistant surgeon in order to achieve an adequate exposure. Excessive point pressure using standard metallic retractors contributes to the compromised sub-dermal arterial plexus.

The circumferential retraction obtained by the use of the Alexis retractor, we believe, eases the point pressure at any specific region of the skin flaps. It also allows for superior views of the operative field. However, in most of our cases, further retraction with standard retractors is still required, albeit with less need for extensive retraction. The device should be viewed as an adjunct to standard retraction rather than its replacement.

Preventative measures to reduce infection include increased use of chlorhexidine for skin preparation and the use of intraoperative breast pocket lavage with antibiotic solutions [9]. However, barrier wound protectors do not appear to have such a firm evidence base in breast surgery as they do in other specialties. We have found this particular retractor to be useful in improving the surgical exposure and in reduction in our wound infection rates. 
Citation: Chaturvedi S, Ramsay G (2013) The Use of a Wound Protector as a Self Retaining Skin Retractor for Skin Sparing Mastectomy and Immediate Reconstruction. Trop Med Surg 1: 112. doi:10.4172/2329-9088.1000112

\section{Conclusion}

Alexis ${ }^{\circledR}$ retractor/wound protector is frequently used in abdominal surgery and is available in many size brackets. We have found this devise very useful in circumferential retraction required for skin sparing mastectomy without point pressure on skin which is common with metallic retractors. Reduction in contamination of the wound is an added advantage.

\section{Conflict of Interest}

The authors declare no potential conflict of interest.

\section{References}

1. Freeman BS (1962) Subcutaneous mastectomy for benign breast lesions with immediate or delayed prosthetic replacement. Plast Reconstr Surg Transplant Bull 30: 676-682

2. Toth BA, Lappert P (1991) Modified skin incisions for mastectomy: the need for plastic surgical input in preoperative planning. Plast Reconstr Surg 87: 10481053.

3. Davies K, Allan L, Roblin P, Ross D, Farhadi J (2011) Factors affecting postoperative complications following skin sparing mastectomy with immediate breast reconstruction. Breast 20: 21-25.

4. Cocquyt VF, Blondeel PN, Depypere HT, Van De Sijpe KA, Daems KK, et al (2003) Better cosmetic results and comparable quality of life after skin-sparing mastectomy and immediate autologous breast reconstruction compared to breast conservative treatment. Br J Plast Surg 56: 462-470

5. Ueda S, Tamaki Y, Yano K, Okishiro N, Yanagisawa T, et al. (2008) Cosmetic outcome and patient satisfaction after skin-sparing mastectomy for breast cancer with immediate reconstruction of the breast. Surgery 143: 414-425
6. Metcalfe KA, Semple J, Quan ML, Vadaparampil ST, Holloway C, et al. (2012) Changes in psychosocial functioning 1 year after mastectomy alone, delayed breast reconstruction, or immediate breast reconstruction. Ann Surg Oncol 19: 233-241.

7. Romics L Jr, Chew BK, Weiler-Mithoff E, Doughty JC, Brown IM, et al. (2012) Ten-year follow-up of skin-sparing mastectomy followed by immediate breast reconstruction. Br J Surg 99: 799-806.

8. Chang RJ, Kirkpatrick K, De Boer RH, Bruce Mann G (2012) Does immediate breast reconstruction compromise the delivery of adjuvant chemotherapy? Breast 22: 64-69.

9. Craft RO, Damjanovic B, Colwell AS (2012) Evidence-based protocol for infection control in immediate implant-based breast reconstruction. Ann Plast Surg 69: 446-450.

10. Edwards JP, Ho AL, Tee MC, Dixon E, Ball CG (2012) Wound protectors reduce surgical site infection: a meta-analysis of randomized controlled trials. Ann Surg 256: 53-59.

11. Cheng KP, Roslani AC, Sehha N, Kueh JH, Law CW, et al. (2012) ALEXIS O-Ring wound retractor vs. conventional wound protection for the prevention of surgical site infections in colorectal resections(1). Colorectal Dis 14: e346-351.

12. Petersen A, Eftekhari AL, Damsgaard TE (2012) Immediate breast reconstruction: a retrospective study with emphasis on complications and risk factors. J Plast Surg Hand Surg 46: 344-348.

13. Carlson GW, Bostwick J 3rd, Styblo TM, Moore B, Bried JT, et al. (1997) Skinsparing mastectomy. Oncologic and reconstructive considerations. Ann Surg 225: $570-575$.

14. Santanelli F, Paolini G, Campanale A, Longo B, Amanti C (2010) The "Type V" skin-sparing mastectomy for upper quadrant skin resections. Ann Plast Surg 65: 135-139. 